\title{
FINITE ELEMENT ANALYSIS AND TEST CORRELATION OF A 10-METER INFLATION- DEPLOYED SOLAR SAIL
}

\author{
David W. Sleight ${ }^{*}$ \\ NASA Langley Research Center, Hampton, VA 23681 \\ Yuki Michii ${ }^{\dagger}$, David Lichodziejewski ${ }^{\dagger}$, and Billy Derbès ${ }^{\dagger}$ \\ L'Garde, Inc., Tustin, CA 92780 \\ Troy O. Mann \\ Swales Aerospace, Hampton, VA 23681 \\ Kara N. Slade and John T. Wang ${ }^{*}$ \\ NASA Langley Research Center, Hampton, VA 23681
}

\begin{abstract}
Under the direction of the NASA In-Space Propulsion Technology Office, the team of L'Garde, NASA Jet Propulsion Laboratory, Ball Aerospace, and NASA Langley Research Center has been developing a scalable solar sail configuration to address NASA's future space propulsion needs. Prior to a flight experiment of a full-scale solar sail, a comprehensive phased test plan is currently being implemented to advance the technology readiness level of the solar sail design. These tests consist of solar sail component, subsystem, and sub-scale system ground tests that simulate the vacuum and thermal conditions of the space environment. Recently, two solar sail test articles, a 7.4-m beam assembly subsystem test article and a 10-m four-quadrant solar sail system test article, were tested in vacuum conditions with a gravity-offload system to mitigate the effects of gravity. This paper presents the structural analyses simulating the ground tests and the correlation of the analyses with the test results. For programmatic risk reduction, a two-prong analysis approach was undertaken in which two separate teams independently developed computational models of the solar sail test articles using the finite element analysis software packages: NEiNastran and ABAQUS. This paper compares the pre-test and post-test analysis predictions from both software packages with the test data including load-deflection curves from static load tests, and vibration frequencies and mode shapes from vibration tests. The analysis predictions were in reasonable agreement with the test data. Factors that precluded better correlation of the analyses and the tests were uncertainties in the material properties, test conditions, and modeling assumptions used in the analyses.
\end{abstract}

\section{Introduction}

Solar sails are examples of ultra-low mass and deployable space structures called gossamer structures. Solar sails have been proposed for a variety of future space exploration missions because they are a cost effective source of propellantless propulsion. Solar sails span very large areas to capture and reflect photons from the Sun and are propelled through space by the transfer of momentum from the photons to the solar sail ${ }^{1,2,3,4}$. Recent advances in gossamer structures will enable a host of useful space exploration missions utilizing solar sail propulsion. Under the direction of the NASA In-Space Propulsion (ISP) Technology Office, the team of L'Garde, NASA Jet Propulsion Laboratory (JPL), Ball Aerospace, and NASA Langley Research Center (LaRC) has been developing a scalable solar sail configuration to address NASA's future space propulsion needs. The $100-\mathrm{m}$ baseline solar sail concept

\footnotetext{
* Aerospace Engineers, Computational Structures and Methods Branch/Research \& Technology Directorate, MS 155, AIAA Members.

${ }^{\dagger}$ Aerospace Engineers, Engineer/Manager/Engineer, AIAA Members.

* Aerospace Engineers, Structural Dynamics Branch/Research \& Technology Directorate, MS 230, AIAA Members.
} 
shown in Fig. 1 was optimized around the 1 astronomical unit (AU) Geostorm mission ${ }^{5,6}$; and features a sail net/membrane with a striped sail suspension architecture with inflation-deployed beams consisting of inflatable sub- $\mathrm{T}_{\mathrm{g}}$ (glass transition temperature) rigidizable semimonocoque booms and a spreader system ${ }^{7,8}$. Sub- $T_{g}$ or cold rigidization takes advantage of the increase in modulus of certain materials below their glass transition temperature. The solar sail has vanes integrated onto the tips of the support beams to provide full three-axis control of the solar sail.

Prior to a flight experiment of a full-scale solar sail, a comprehensive phased test plan is currently being implemented to advance the technology readiness level of the solar sail design ${ }^{7,8}$. The tests consist of solar sail

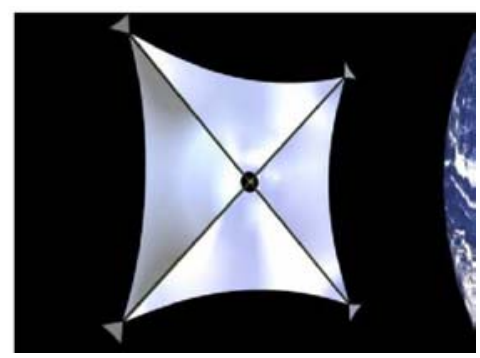

Figure 1. L'Garde 100-m ISP solar sail design.

component, subsystem, and sub-scale system ground tests that simulate aspects of the space environment such as vacuum and thermal conditions. Due to the large size and ultra-low mass of solar sails, the influence of gravity makes full-scale structural response measurements via ground tests difficult and unrepresentative of the space environment. A suspension system was used to offload the structure to mitigate the effect of gravity. A key to the maturation of solar sail technology is the development of validated finite element analysis (FEA) models that can be used for design and analysis of solar sails. A major objective of the effort is to utilize the available ground test data to validate the nonlinear finite element methods and models simulating the ground tests. This paper presents a correlation of the structural analyses with the ground test data. Comparisons are performed for pre-test and post-test analysis predictions with the ground test data including load-deflection curves from static load tests, and vibration frequencies and mode shapes from vibration tests.

\section{Ground Test Program}

The comprehensive phased ground test program consists of three phases for testing the solar sail system in a relevant environment for advancing the Technology Readiness Level (TRL) to a level of nearly 6 (system model demonstrated/validated in relevant environment) in 2005. The ground tests consist of solar sail component, subsystem, and sub-scale system test articles based on the inner portions of the $100-\mathrm{m}$ baseline solar sail design. Initially, boom components of the solar sail system were tested to determine material and section properties. Next, subsystem test articles of the solar sail system were tested. A subsystem structural ground test was performed at the NASA Goddard Space Flight Center (GSFC) Thermal Vacuum Chamber on a deployed 7.4-m beam assembly consisting of the inflation-deployed boom, a spreader system, and a gravity-offload system as shown in Fig. 2. Test conditions included deployment, combined solar flux loading with bending, combined solar flux loading with torsion, and beam structural vibration tests. The next phase of the program was the testing of sub-scale solar sail system test articles. The first sub-scale solar sail system test was performed in vacuum environment conditions in July 2004 at the Space Power Facility (SPF), located at the NASA Glenn Research Center (GRC) Plum Brook Station. The 10-m scaled solar sail test article consisted of a four-quadrant sail net/membrane with the solar sail
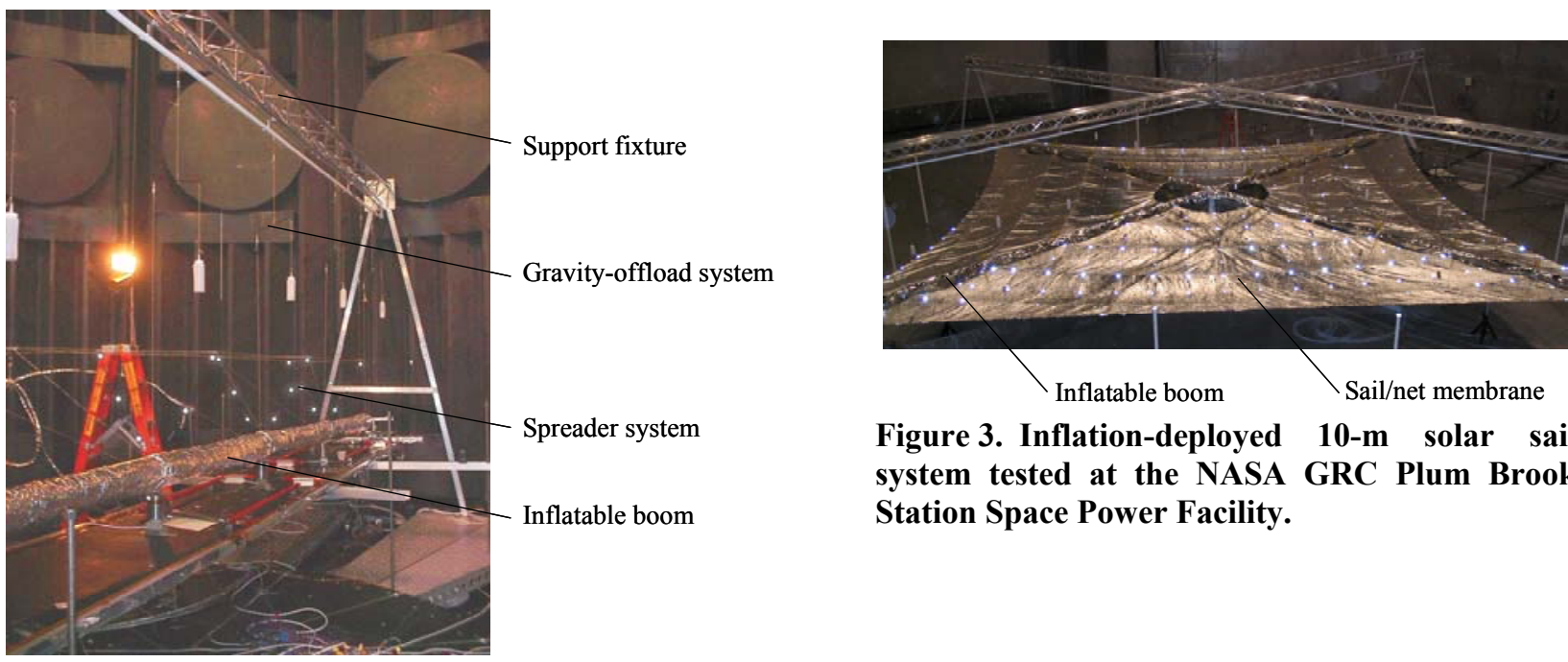

Figure 3. Inflation-deployed 10-m solar sail system tested at the NASA GRC Plum Brook Station Space Power Facility.

Figure 2. 7.4-m beam assembly at the NASA GSFC

Thermal Vacuum Chamber. 
beam assemblies as shown in Fig. 3. Test conditions included deployment, vertical and horizontal bending, torsion, and beam/membrane structural vibration tests. The final scaled solar sail system test will be a 20 -m four-quadrant solar sail test article to be tested at the SPF in July 2005. Both system tests will advance the technology level needed for a solar sail flight demonstration.

\section{Structural Analysis and Ground Test Correlation}

For programmatic risk reduction, a two-prong analysis approach was undertaken. Two separate teams developed computational models of the solar sail test articles using different FEA software packages. L'Garde used NEiNastran ${ }^{9}$, while LaRC used ABAQUS ${ }^{10}$ in the analysis effort. Each team independently developed computational models of the solar sail test articles in incremental stages for the multi-phased program. With each stage of the program, the computational models increased in complexity from simple component models to highfidelity system-level models. The goal of the structural analysis effort of the 7.4-m beam assembly and the $10-\mathrm{m}$ sub-scale solar sail system was to develop FE models and perform analyses that adequately predicted the response of the ground tests. The models were developed based on the cumulative test and analysis correlation results of previous component and sub-scale tests. An important function of the structural analyses was to provide pre-test predictions of the expected structural response that was needed to help plan the tests. After the tests, efforts were performed to improve the test and analysis correlation. The models will be modified and used to predict and plan for the upcoming 20-m solar sail test at the NASA GRC Plum Brook Station. The following sections describe the ground test articles and testing, the finite element modeling and structural analysis details, and the correlation of the ground test and structural analysis results for the two solar sail test articles tested in the ISP program.

\section{A. Beam Assembly Ground Test and Analysis}

\section{Beam Assembly Test Article and Testing Description}

The 7.4-m beam assembly subsystem test article shown in Fig. 2 was tested in the NASA GSFC Thermal Vacuum Chamber. The beam assembly design is a combination of two elements: the inflatable sub- $\mathrm{T}_{\mathrm{g}}$ rigidizable boom and the sun-side spreader system. The inflatable sub- $\mathrm{T}_{\mathrm{g}}$ rigidizable boom is the primary load carrying member, and the sun-side spreader system reinforces the boom against the bending moment imparted by the solar flux on the sail membrane. The booms are designed in a semi-monocoque configuration (See Fig. 4) with high modulus fibers that are impregnated with a sub- $\mathrm{T}_{\mathrm{g}}$ resin to rigidize the structure after deployment. The spreader system shown in Fig. 5 incorporates a number of tensioned lines running along the boom. The anchor lines running from the spreader tips to the rings provide a shear component to transmit the bending loads. The longeron lines running along the spreader tips provide a large moment of inertia to the boom to resist the bending generated by the solar flux loading. The diagonals, which cross over along alternating spreader tips, increase the torsional stiffness of the truss while providing longitudinal redundancy in the lines in case one or more lines are severed. The battens, which are connected between the spreader bars, are pre-tensioned to a specified force during the construction. The spreader bars are a thin-walled circular composite construction.

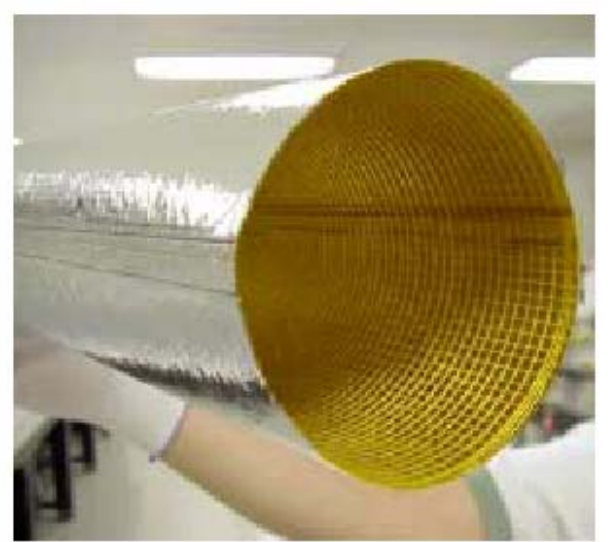

Figure 4. Semi-monocoque boom design ${ }^{8}$.

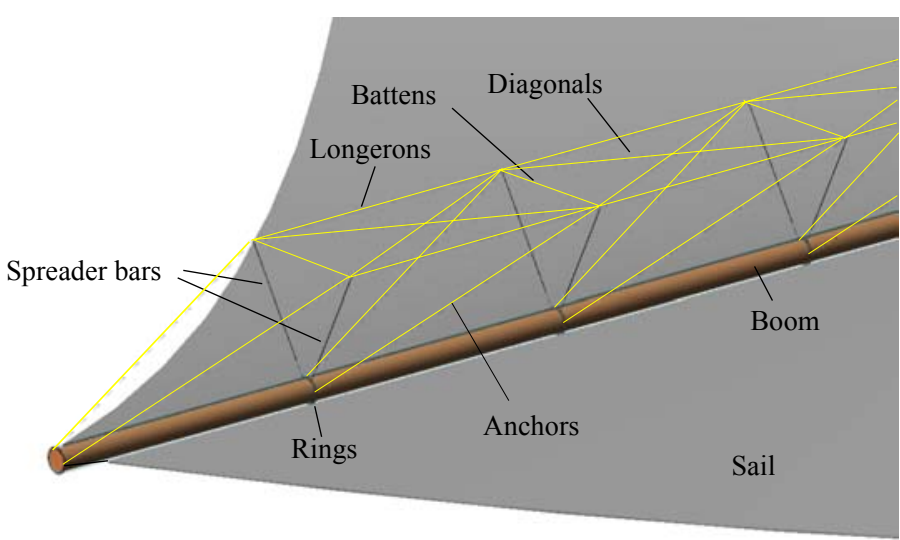

Figure 5. Spreader system of beam assembly.

Tests were designed to demonstrate the structural and rigidization of the beam assembly at operational conditions. Cold plates placed directly below the booms simulate an environment where the booms would rigidize by the cold rigidization process. A test to simulate a solar flux loading condition consisted of a combination of shear, 
compression, and moments applied to the beam assembly as shown in Fig. 6. The applied loads were sized to simulate the highest anticipated loads at the root of the 100-m full-scale baseline solar sail design. The combined loads test set-up is shown in Fig. 7. The test article was offloaded for gravity at multiple locations along the booms to simulate the zero-g space environment. Masses were hung on an overhead balance bar attached to the booms and a support fixture with thin strings. The gravity-offloaded masses were adjusted to level the booms. Masses and moment arms at the boom tip simulated the various flight loads conditions on the boom. The beam assembly was cantilevered at the base of the boom tube. The lines connected to the first two spreader bars (called the cat's cradle) were secured for additional constraints.

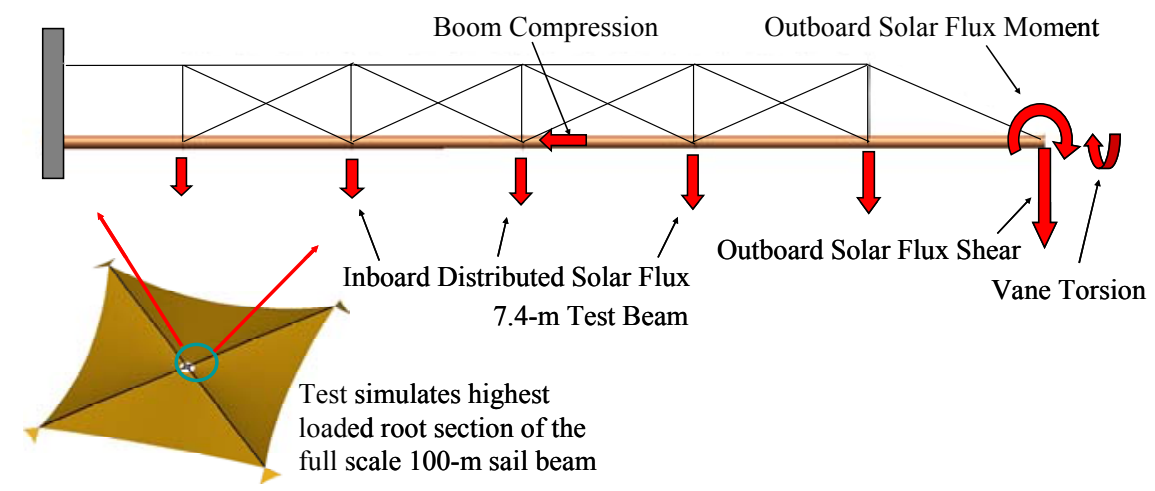

Figure 6. Simulated operational loading conditions for the beam assembly test.

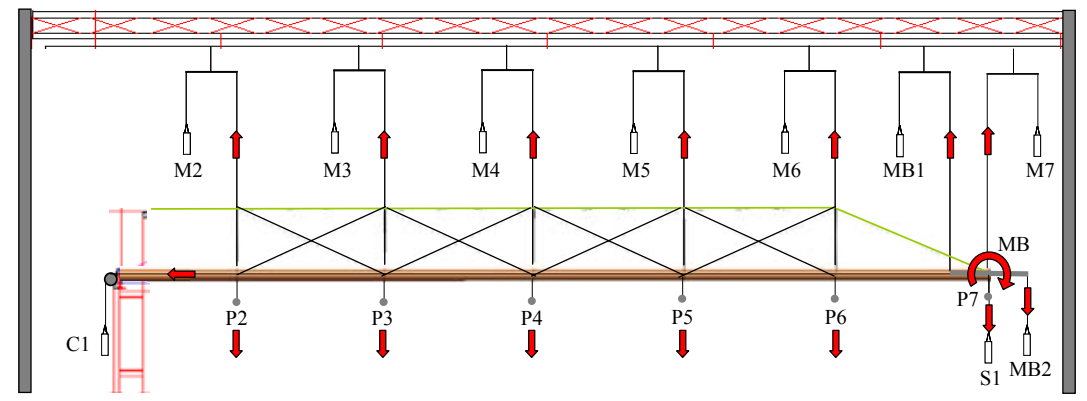

Figure 7. Beam assembly test set-up.

Structural testing was conducted at nominal operating conditions at 0.1 torr $(13.3 \mathrm{~Pa})$ vacuum pressure and $-70^{\circ}$ $\mathrm{F}\left(-56.7^{\circ} \mathrm{C}\right)$ on the beam assembly test article. Two static structural tests were performed on the beam assembly. In the first test, a combined solar flux loading and vertical bending test was conducted to demonstrate that the beam assembly could withstand solar loads in a relevant environment. In the second test, a combined solar flux loading and torsion loading condition was applied to demonstrate that the beam assembly withstand vane torsion loads in a relevant environment. A stepper motor was used to apply the shear loads in the bending test and the moment to simulate the vane actuator forces in the torsion test. Photogrammetry and proximity sensors were used to measure the deflections, and load cells were used to measure the loads. A structural vibration test was also performed on the beam assembly for the solar flux loading condition. An electromagnetic actuator and controller excited the modes of the beam assembly. An accelerometer was mounted to the beam assembly to measure the vibration data. Unfortunately, data were not collected due to equipment failure during testing. Video data were used to alternatively determine the frequencies. The test results are presented in the following section.

\section{Beam Assembly Modeling and Test/Analysis Correlation}

Finite element models of the 7.4-m beam assembly were developed based on the specifications of beam assembly test article. Figure 8 shows the NEiNastran and ABAQUS finite element models used to simulate the beam assembly test. Both models were based on the same geometry, and used beam elements to model the solar sail boom and spreader bars, and truss or cable elements to model the spreader system lines. To model the cable behavior in 
ABAQUS, the *NO COMPRESSION material behavior option was included in the analysis. The rings on the boom and other test masses measured during the test were modeled as concentrated masses in the models. In addition, rigid elements were used in NEiNastran and multi-point constraints (MPC's) were used in ABAQUS to model the connection of the spreader bars to the rings on the boom. The gravity-offload system was not explicitly modeled in either FEA software packages because some of the details of the test set-up were not available until after the test. Point forces were used to apply equivalent forces of the weight instead of explicitly modeling the cables, bars, and masses of the gravity-offload system. The material properties in the boom and spreader bars were based on measured component test results, while the material properties of the spreader system lines were based on nominal properties from the manufacturer. The base of the boom was fixed and ends of the lines of the cat's cradle, located at the hub of the solar sail (See Fig. 8 (a)) were pinned

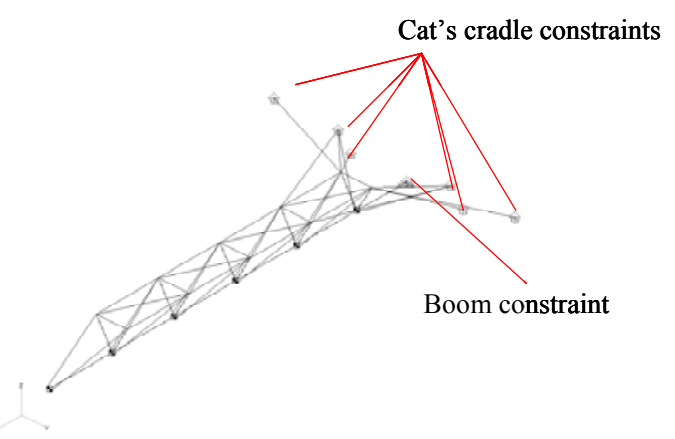

(a) NEiNastran model.

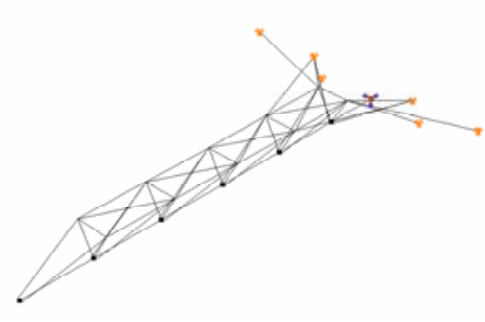

(b) ABAQUS model.

Figure 8. Finite element models of 7.4-m beam assembly test article.

Geometrically nonlinear analyses were performed by both teams to analyze the beam assembly test article. A similar loading scheme was used by both teams. First, a pre-tensioning load was applied to the battens in the model combined with a uniform gravity load on the test article. Second, point forces were applied to each ring location to simulate the gravity-offloading of the test article. The gravity-offload forces at the ring locations were adjusted until the deflection of the boom was nearly zero. Finally, loads were applied at the boom tips to simulate the appropriate test loading condition as shown in Fig. 6. The test loading conditions included a combined solar flux loading and vertical bending (shear) loading condition, a combined solar flux loading and torsional loading condition, and vibration under a solar flux loading condition.

In the combined solar flux loading and vertical bending test, a stepper motor was used to apply a specified boom tip deflection, and load cells were used to measure the resulting force. The deflections were calculated based on the number of revolutions of the stepper motor. The vertical bending load as a function of the boom tip vertical displacement is shown in Fig. 9 for the test data, pre-test analytical predictions, and post-test correlated analyses. The vertical bending load was normalized by the maximum measured force, and the boom tip displacement was normalized by the maximum applied vertical displacement. The pre-test predictions from both FEA software packages that used the original properties were close to the test data as shown in Fig. 9. The scatter of the test data for deflections less than a normalized boom tip deflection of 0.6 was attributed to the use of the stepper motor at the beginning of the test and inaccuracies in the calculation of the displacement. After the test, additional analyses were performed in which the properties of the spreader system lines on the beam assembly were modified to bring the FEA results closer to the test data.

In the combined solar flux loading and torsion test, the rotation of the boom tip from the test data was much greater than the FEA predictions. However, the boom tip test data included had a small deflection in the vertical direction. This deflection indicates that a combined vertical loading and torsional loading condition may have been applied in the test. A comparison of the test data and FEA predictions is not included here until further investigation is performed on the test data. Results from both FEA software packages compared well to each other in pre-test and post-test analyses for the pure torsion loading condition. 


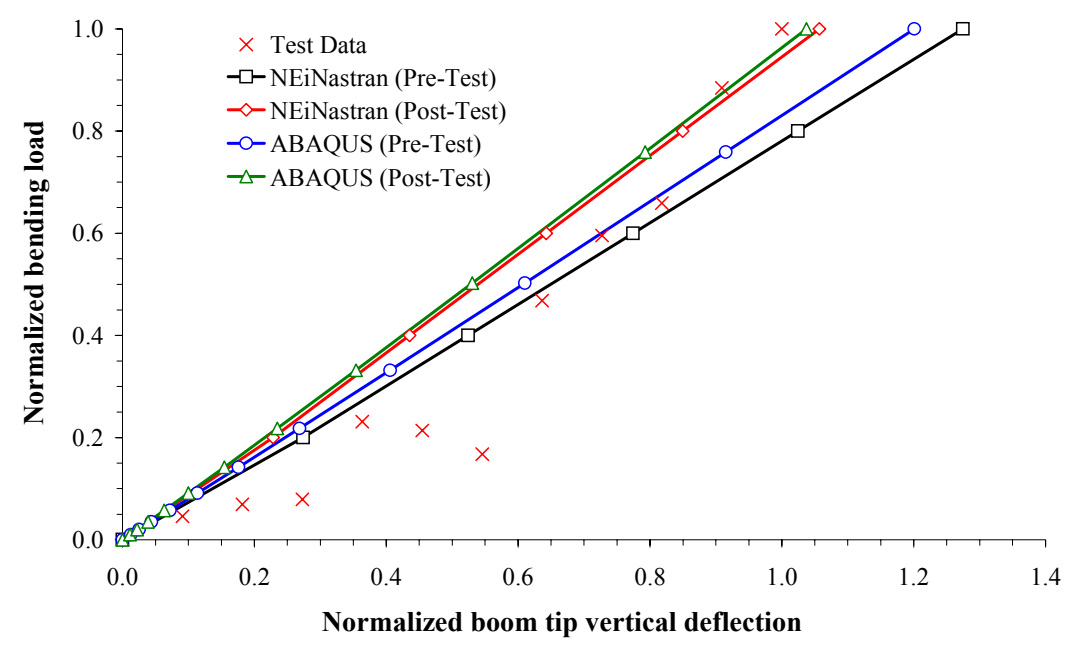

Figure 9. Load vs. deflection curve for beam assembly vertical bending test.

A structural vibration test was also performed on the beam assembly to determine the frequencies and mode shapes for the lateral and vertical bending modes. The mode shapes predicted by both FEA software packages showed qualitative agreement with the lateral and vertical bending modes of the test based on observations of videos of the test. The lateral and vertical bending mode shapes predicted by the ABAQUS FEA are shown in Fig. 10. The mode shapes from the NEiNastran analysis also were similar to the ABAQUS analysis results. A comparison of the frequencies for the test data and analyses are listed in Table 1. The frequencies were estimated using videos of the vibration test because the accelerometer debonded from the beam assembly. This debonding was attributed to the low temperatures of the test. The frequency predictions for both FEA software packages compared well with each other, but both analyses over-predicted the test frequencies. Examination of the strain energies in the analysis indicated that the tensioned lines of the cat's cradle region were the biggest contributor to the vibration modes. Conditions from the test set-up that were not explicitly modeled may have contributed to differences between the FEA predictions and the test results. For example, the amount of slackness or tension in the spreader system lines was unknown in the test, and was neglected in the models. Moreover, ignoring the gravity-offload system in the models also contributed to the discrepancies between the test and predicted frequencies. An analysis was later performed in ABAQUS with the gravity-offload system included in the model. The analysis predicted the same first frequency as the previous analysis; however, the second frequency decreased from $1.99 \mathrm{~Hz}$ to $1.42 \mathrm{~Hz}$.

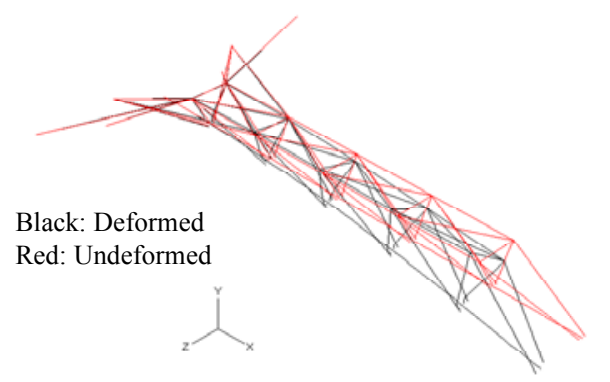

(a) Mode 1: Lateral bending mode

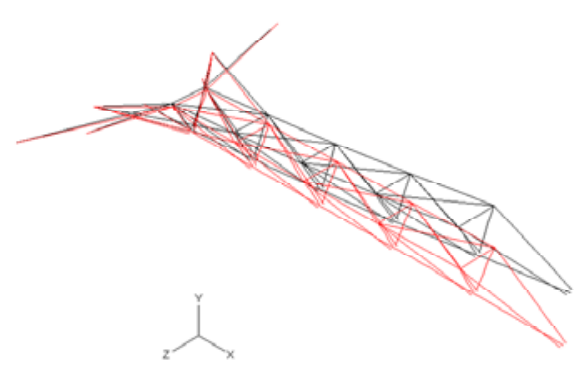

(b) Mode 2: Vertical bending mode

Figure 10. Mode shapes predicted by ABAQUS for beam assembly test article. 
Table 1. Comparison of test and FEA frequencies for beam assembly structural vibration test.

\begin{tabular}{|c|c|c|c|}
\hline Mode & $\begin{array}{c}\text { Test } \\
\text { Frequency }(\mathrm{Hz})\end{array}$ & $\begin{array}{c}\text { NEiNastran } \\
\text { Frequency }(\mathrm{Hz})\end{array}$ & $\begin{array}{c}\text { ABAQUS } \\
\text { Frequency }(\mathrm{Hz})\end{array}$ \\
\hline 1 & 0.60 & $0.86(+43.3 \%)$ & $0.92(+53.3 \%)$ \\
\hline 2 & 0.80 & $1.79(+123.8 \%)$ & $1.99(+146.8 \%)$ \\
\hline
\end{tabular}

\section{B. Ten-Meter Four-Quadrant Solar Sail System Test and Analysis}

1. Ten-Meter Solar Sail System Test Article and Testing Description

A 10-m four-quadrant solar sail system test article, fixed at the center for all translations (X, Y, Z) and rotations $\left(R_{x}, R_{y}, R_{z}\right)$, was tested in two configurations in the SPF in vacuum conditions at the NASA Glenn Plum Brook Station in July $2004^{11}$. The first test configuration, shown in Fig. 3, was a sail membrane configuration with four sail/net membrane quadrants attached to the rings on the booms. A similar gravity-offloading scheme employed in the beam assembly subsystem test was used for the 10-m sail membrane assembly configuration. Cold plates beneath the booms were used to rigidize the booms. This configuration was used to measure the static sail membrane shape and vibration frequencies under a combined simulated solar flux loading and gravity loading condition as shown in Fig. 11. The simulated solar flux loading was applied to the tips of all four beams by adding masses to the gravity-offload system to apply solar flux forces denoted as $F_{1}$ and $F_{2}$ in Fig. 11. The static sail membrane shape was measured by photogrammetry. The targets used in the photogrammetry can be seen in Fig. 3 as the illuminated dots on the sail membrane. For the sail membrane structural vibration test, the excitation was provided by two electromagnets placed at the beam tips, where one magnet excited the structure laterally, and the other one excited the structure vertically. The natural frequencies and mode shapes of the sail membrane were measured by laser vibrometry.

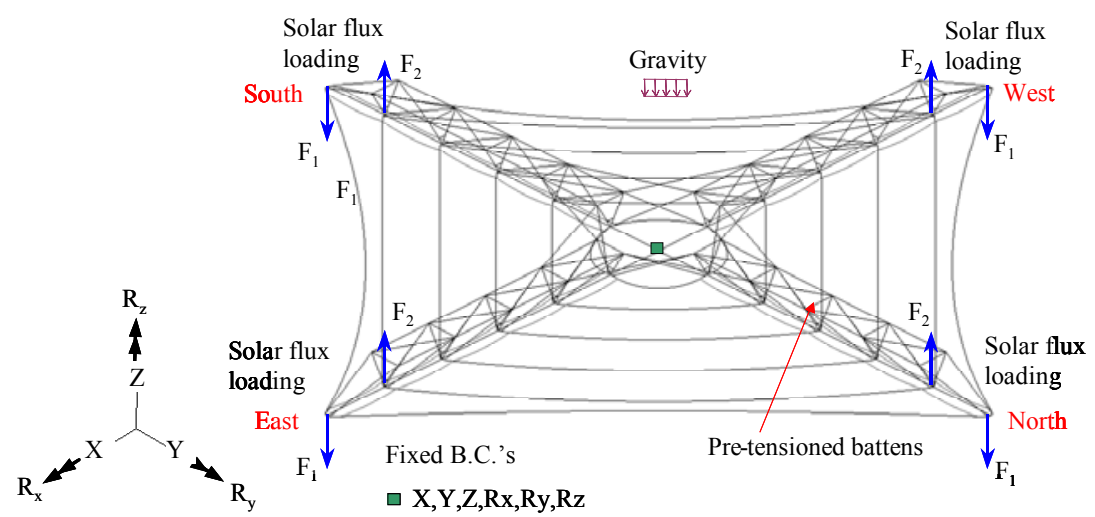

Figure 11. Loading condition for sail membrane configuration test.

The second configuration tested in the SPF was the beam structural assembly with the sail membrane quadrants replaced with lightweight strings located between the last four ring stations on the beam assemblies as shown in Fig. 12. The strings were chosen such that the weight of the strings in a 1-g gravity field would represent the solar pressure imparted on the sail during operation. The test article was also gravityoffloaded in this configuration. A structural vibration test and three static load-deflection tests were performed in vacuum conditions. The structural vibration test measured the natural frequencies and mode shapes about a solar flux and gravity loading condition with accelerometers

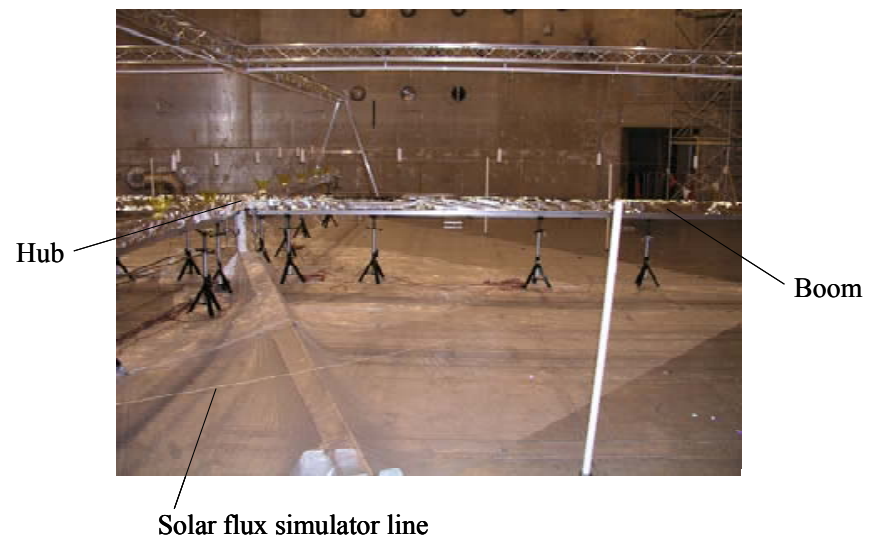

Figure 12. 10-m sail system test article for beam structural assembly static loads and vibration tests. 
placed along the length of the booms. The loading and boundary conditions for the structural vibration test are given in Fig. 13 (a). Several of the ring locations on the booms were tied to the cold plates to introduce fixed boundary conditions at these locations as denoted by the triangles in the figure. As stated earlier, the center of the solar sail was fixed in all directions. The static load-deflection tests were performed on the North boom of the structure (see Fig. 11) in the SPF to measure the deflection of the boom in horizontal and vertical bending and torsion along with the solar flux and gravity loading condition. The loading and boundary conditions for the static load-deflection tests are shown in Figs. 13 (b)-(d). Actuators attached to the boom tip allowed the load-displacement tests to be performed.

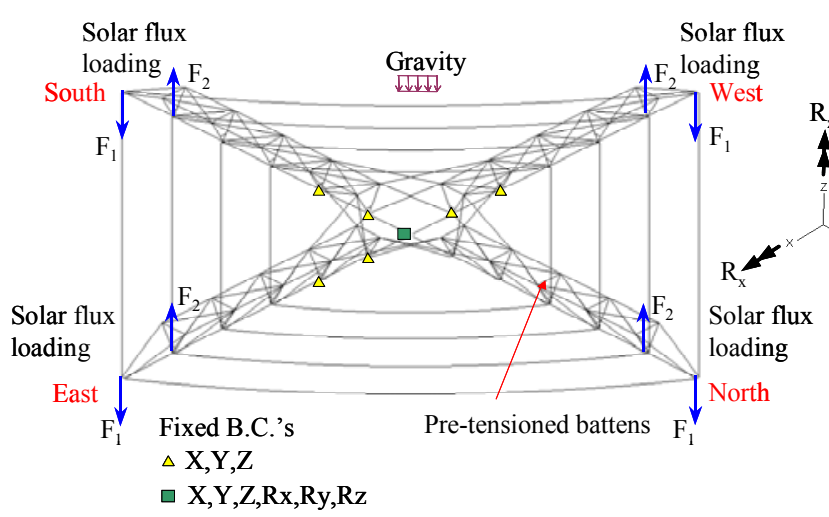

(a) Loading and boundary conditions for structural vibration test

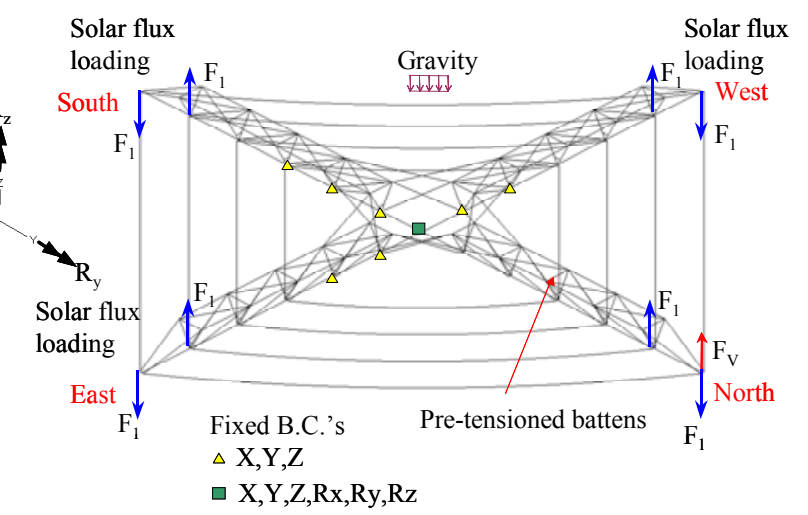

(b) Loading and boundary conditions for vertical bending test

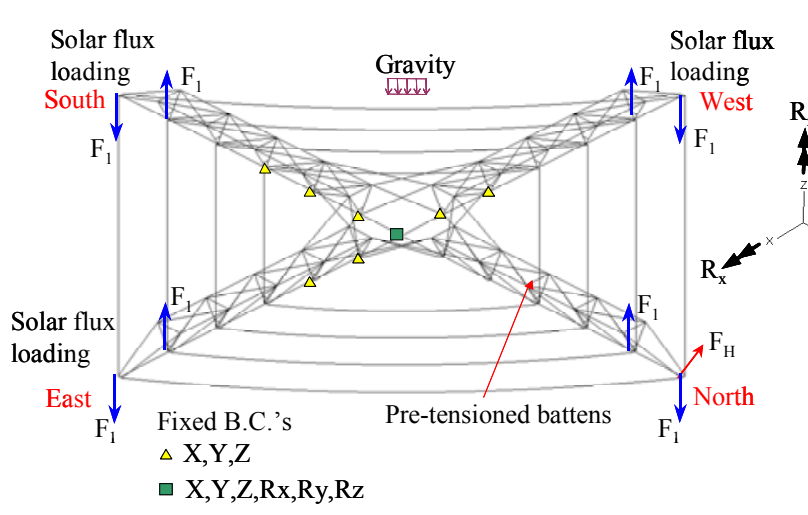

(c) Loading and boundary conditions for horizontal bending test

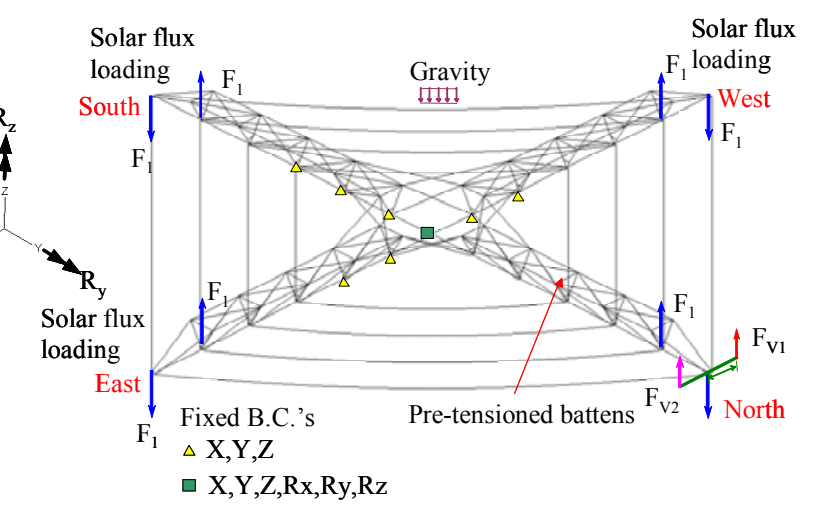

(d) Loading and boundary conditions for combined vertical bending and torsion test

Figure 13. Loading and boundary conditions for beam structural assembly tests.

\section{Ten-Meter Solar Sail Membrane Configuration Modeling and Test/Analysis Correlation}

Both teams analyzed the 10-m solar sail membrane configuration to compare to the sail static shape and sail membrane vibration test measurements. Figures 14 (a) and (b) show the L'Garde NEiNastran and the NASA LaRC ABAQUS finite element models of the 10-m solar sail system test article, respectively. The L'Garde NEiNastran model utilized shell elements for the sail membrane, beam elements for the booms and spreader bars, and tensiononly cable elements for the spreader system lines in the beam assemblies. The LaRC ABAQUS model utilized membrane elements for the sail membrane, beam elements for the booms and spreader bars, and truss elements for the spreader system lines in the beam assemblies. Both models included concentrated mass elements to account for added masses measured during the test set-up. The gravity-offload system was not modeled by either team. 


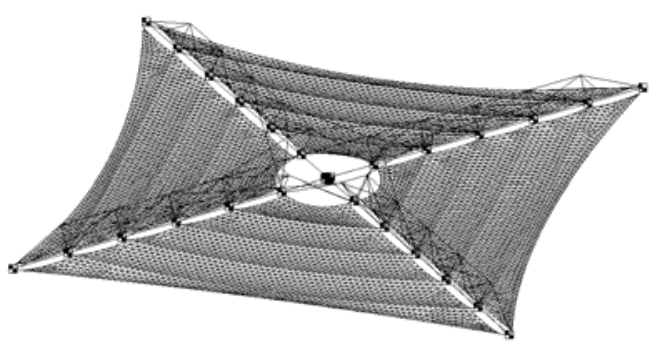

(a) NEiNastran model.

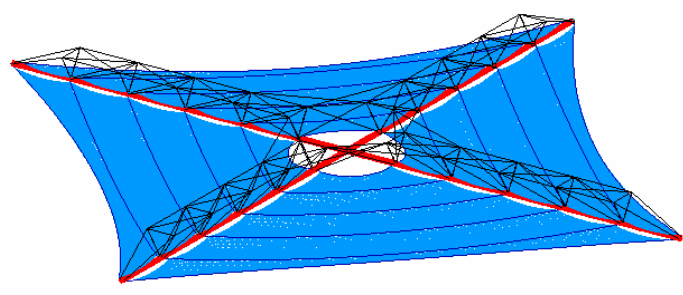

(b) ABAQUS model.

Figure 14. Finite element models of 10-m solar sail system sail test article for the sail membrane configuration.

The 10-m solar sail test article with its ultra-thin sail membrane and long slender booms presented many analysis challenges due to its extreme geometrically nonlinear behavior. Geometrically nonlinear analyses were performed with NEiNastran and ABAQUS. Adjusting various nonlinear solution parameters aided the convergence of the NEiNastran analysis. However, convergence was not obtained for the sail membrane vibration analysis using shell elements. Instead, an alternate NEiNastran isogrid model was developed in which bar elements were used to model the sail membrane. In ABAQUS, the stabilization feature was employed to achieve convergence in the analysis. The stabilization feature provides a mechanism for stabilizing unstable quasi-static problems through the addition of volume-proportional damping forces to the equations of motion. Multiple load steps were used in both the NEiNastran and ABAQUS analyses. First, a pre-tensioning load was applied to the battens combined with a uniform gravity load on all elements in the models. Second, point forces were applied to each ring location equivalent to the force resultant of the overhead masses on the gravity-offload system. Third, the forces in Fig. 11 were applied to the appropriate boom locations to simulate the solar flux loading condition. Finally, the vibration frequencies and mode shapes about the nonlinear stressed state were determined.

Figure 15 shows a comparison of the 10-m sail membrane out-of-plane static shape for the two FEA software packages and the photogrammetry test data. The predicted static out-of-plane shapes were determined by adding the out-of-plane deformations to the original undeformed geometry of the finite element models. The contour plots show that maximum billow of the sail occurs at the center of each sail quadrant near the outer edges. Both FEA software packages under-predicted the sail billow as compared to the photogrammetry test data. The differences between the analyses and the test may be attributed to uncertainties in the stiffness in the spreader system lines as will be described in the next section. Also, sail wrinkling that was not accounted for in the analyses could also contribute to the shape difference between the test and the analyses.

For the sail membrane structural vibration test, a comparison of the first three sail membrane frequencies in the vacuum environment is presented in Table 2 for the test and the analyses. The FEA mode shapes were selected based on the resemblance to the modes shapes of the test. Both FEA software packages showed good agreement with the measured test frequencies and mode shapes. Figure 16 shows a comparison of the first mode shape for the test data using laser vibrometry of the Northeast sail membrane quadrant and the FE analyses. The Northeast sail membrane quadrant is identified by the symbol "A" in Fig. 16.

Table 2. Comparison of test and FEA frequencies for 10-m sail membrane structural vibration test.

\begin{tabular}{|c|c|c|c|}
\hline Mode & Test Frequency $(\mathrm{Hz})$ & NEiNastran Frequency $(\mathrm{Hz})$ & ABAQUS Frequency $(\mathrm{Hz})$ \\
\hline 1 & 1.10 & $1.31(+19.1 \%)$ & $1.25(+13.6 \%)$ \\
\hline 2 & 3.25 & $3.29(+1.2 \%)$ & $2.85(-12.3 \%)$ \\
\hline 3 & 7.55 & $7.22(-4.4 \%)$ & $7.33(-2.9 \%)$ \\
\hline
\end{tabular}




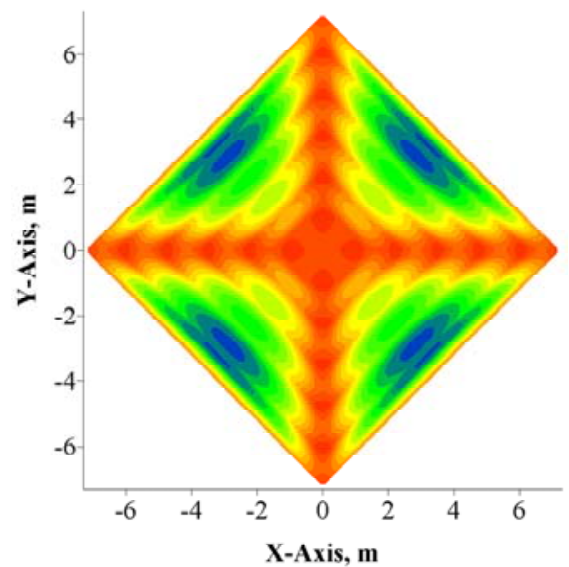

(a) NEiNastran analysis

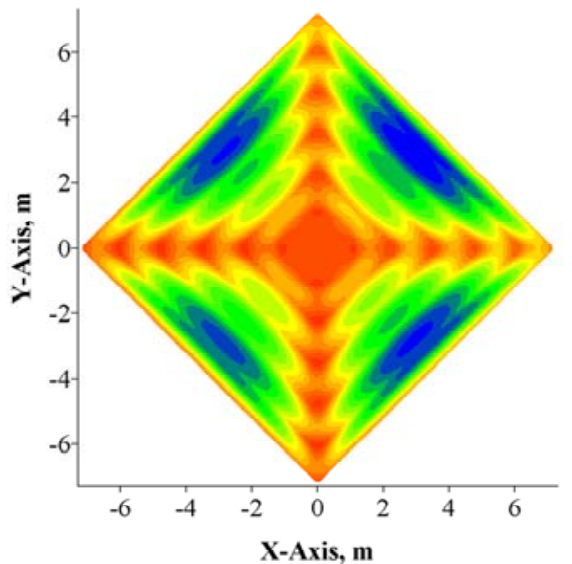

(b) ABAQUS analysis

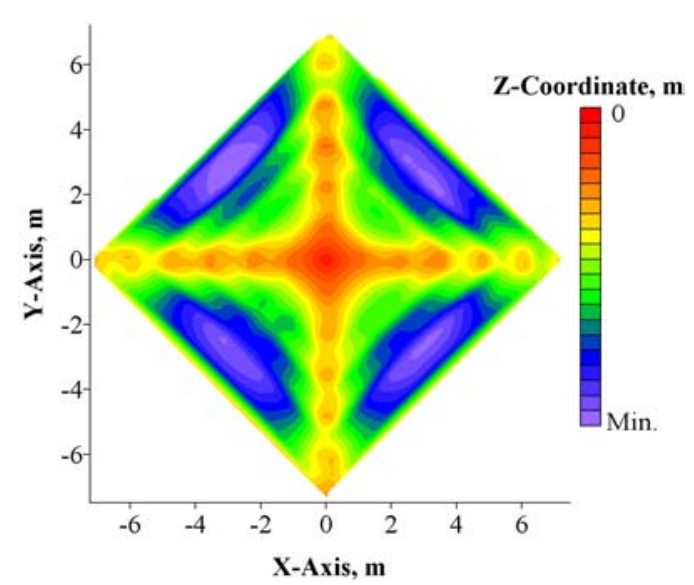

(c) Photogrammetry test data

Figure 15. 10-m sail membrane static shape comparison for FEA and test results. 


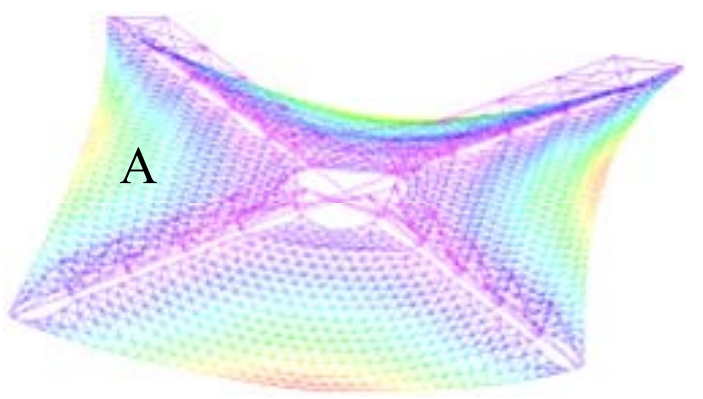

(a) NEiNastran analysis

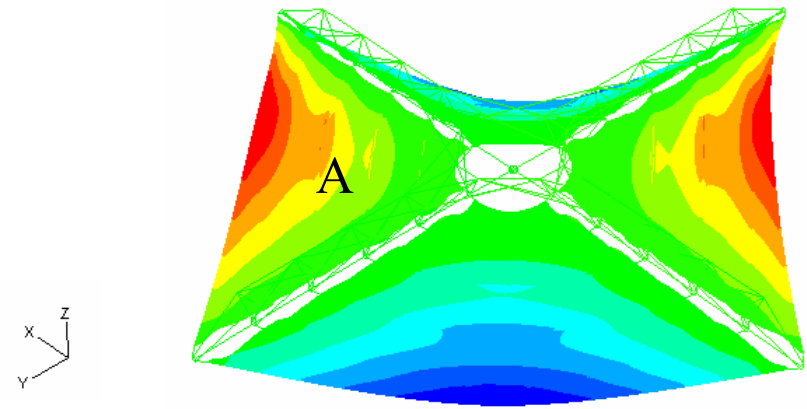

(b) ABAQUS analysis

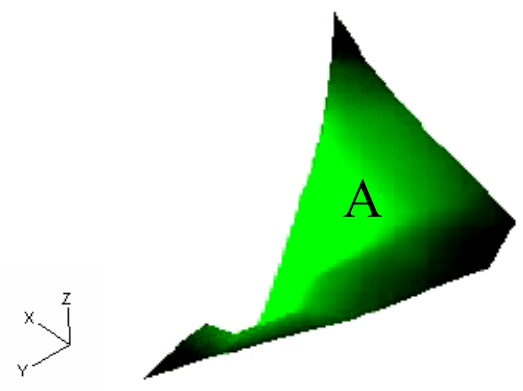

(c) Sail membrane vibration test data

Figure 16. First mode shape comparison for 10-m sail membrane vibration test and analysis results.

\section{Ten-Meter Solar Sail Beam Structural Assembly Configuration Modeling and Test/Analysis Correlation}

In the second test of the $10-\mathrm{m}$ solar sail test article, the sail membrane quadrants were removed and replaced with lightweight strings to simulate a solar flux loading condition in the space environment. The weight of the strings in a 1-g gravity field was equivalent to a solar pressure load imparted on the sail during operation. One primary objective of the test was to measure the beam structural assembly static deflections subjected to a vertical bending load case, a horizontal bending load case, and a combined vertical bending/torsion load case. Another objective was to measure the dynamics of the beam structural assembly including natural frequencies and mode shapes. Finite element models were developed in NEiNastran and ABAQUS specifically to model this test configuration. Multiple load steps were used in the analyses as previously described. However, in the last load step, the test load conditions were applied to the beam assemblies to simulate the appropriate bending, torsion, and dynamic test cases.

The vertical bending test was conducted on the beam structural assembly to apply the vertical bending loads with linear actuators. Unfortunately, the position of the boom following the cold rigidization process did not allow for the displacements to be applied downward as originally planned due to clearance issues with the cold plates. As a consequence, the vertical bending test was performed by applying loads in the upward direction to the North beam as shown in Fig. 13 (b). These loads were not representative of the operational loads of the sail structure. The loaddeflection curve for the vertical bending test is presented in Fig. 17 for the test data and the FE pre-test and post-test analyses. The vertical bending load was normalized by the maximum vertical bending force, and the boom tip displacement was normalized by the maximum vertical deflection from the test. The test data showed two distinct linear regions in the response of the beam. The first region, up to a normalized displacement value of 0.30 , most likely represents the stiffness of the beam structural assembly. The second region most likely represents the stiffness of the boom only as the spreader system lines go slack as the beam structural assembly was deflected upward. The pre-test FE analyses using NEiNastran and ABAQUS did not correlate very well with the test data. Particular factors altering the structure contributed significantly to the differences between the FEA predictions and the test results. One significant factor identified during the test was a significant reduction in the stiffness of the spreader system lines as a result of coloring the lines with markers for test set-up identification. The saturation of the ink on the spreader system lines reduced their stiffness by a factor or three or more according to tension tests of the colored 
lines conducted after the Plum Brook test. The stiffness loss in the spreader system lines also varied by the color of the marker used to mark the lines. Another factor was the change in the direction of the applied displacement to allow for equipment clearance. The combination of the reduced stiffness of the lines and the change in loading direction of the beam assembly caused the spreader system lines in the beam assemblies to become slack. Consequently, the test results show much larger deflections than the analyses predicted.

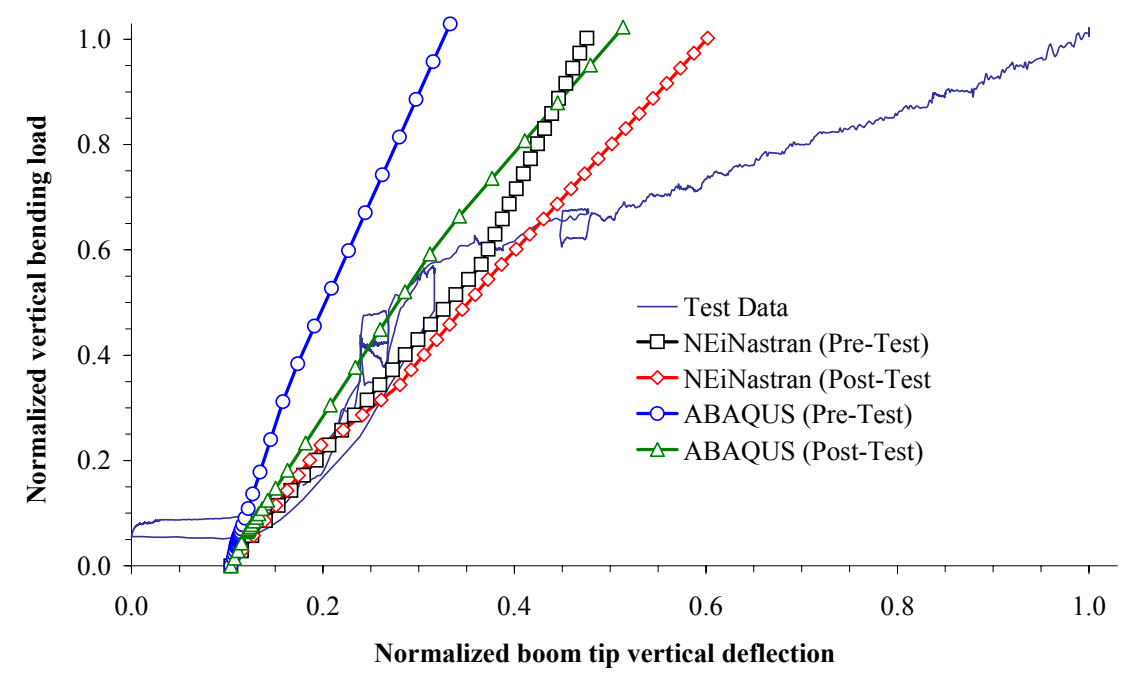

Figure 17. Vertical bending test and analysis results for $10-\mathrm{m}$ beam
structural assembly static load-deflection test.

The horizontal bending test and FEA predictions are presented in Fig. 18. The horizontal bending load was normalized by the maximum horizontal bending force, and the horizontal boom tip deflection was normalized by the maximum horizontal deflection from the test. The horizontal bending loads were applied to the sail beam with single linear actuators placed on either side of the beam. The initial intent was to measure the deflection of the beam in both bending directions to determine any biases in the structure. However, due to the deflection of the beam (and potentially the cold plate incident mentioned earlier), the beam assembly moved out of range of one of the linear actuators during the test, and therefore a horizontal bending load in only one direction was recorded. The FE analysis results predicted a much stiffer structure than the test results indicated. A contributing factor may have been asymmetries in the amount of tension or slack in the spreader system lines of the beam assemblies. A separate analysis was performed on the boom tube without the spreader system in NEiNastran, and in that case, the predicted load-deflection curve matched the test data.

The torsion test of the beam structural assembly was supposed to be performed by applying equal and opposite loads to either side of the torsion bar attached to the boom tip. However, the lower actuators were out of range of the beam after it cooled. As a result, only one vertical load was applied on the ends of the torsion bar (shown in green in Fig. 13 (d)). Thus, the applied load was then a combination of vertical bending and torsion. Two tests were performed by applying vertical forces of $\mathrm{F}_{\mathrm{V} 1}$ in the first test and $\mathrm{F}_{\mathrm{V} 2}$ in the second test. The combined vertical bending/torsion static test and FEA predictions are presented in Fig. 19. The vertical bending load was normalized by the maximum vertical bending force and the vertical boom tip deflection was normalized by the maximum vertical deflection from the test. The load-deflection curve shows results for two loading torsion directions which may be attributed to the different tension or slack states in the spreader system lines because of the loss in stiffness due to the ink markings. Both FEA software packages predicted different results for the pre-test analyses which may be attributed to the assumptions used in the model development and analysis. 
Both FE models were updated following the test to improve the test correlation. Updates to the models included lowering the modulus of the spreader system lines in the beam assemblies to account for the coloring of the lines and lowering the modulus of the boom based on boom component test results. The FEA predictions with the reduced properties are shown in Figs. 17-19. The correlation among the FEA predictions and the test data improved for the vertical bending and torsion test cases. However, the analysis results with the updated properties did not improve sufficiently for the horizontal bending case. In general, the analyses indicated that the stiffness of the spreader system lines in the beam assemblies affected the response of the 10-m beam structural assembly. As the amount of tension or slackness in the spreader system lines in the test was not measured, close test/analysis correlation is precluded.

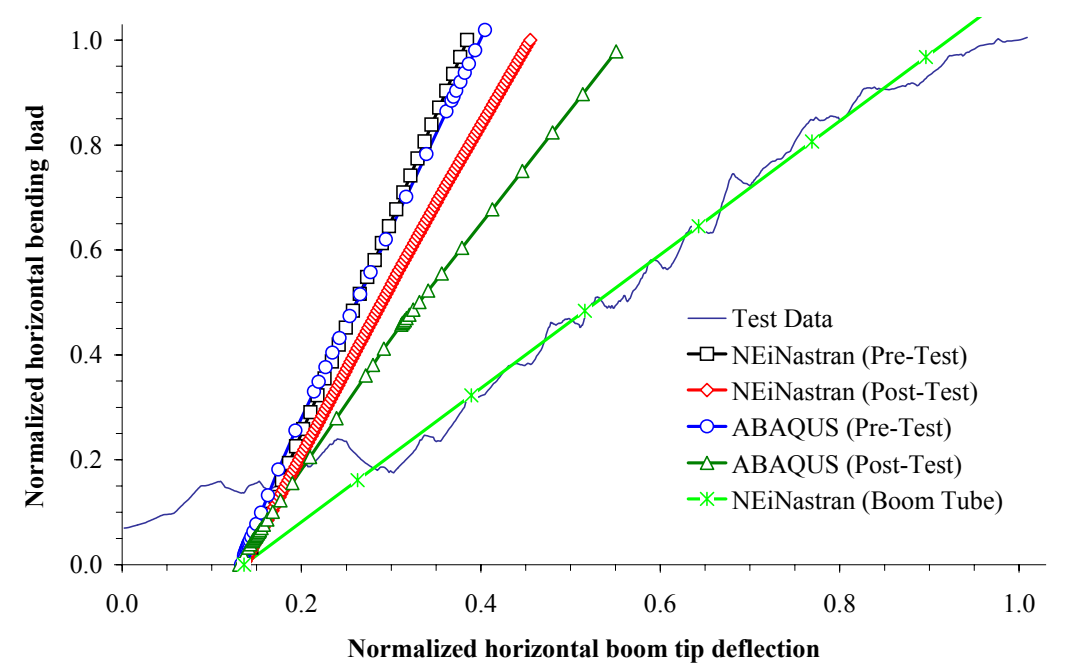

Figure 18. Horizontal bending test and analysis results for $10-\mathrm{m}$ beam structural assembly static load-deflection test.

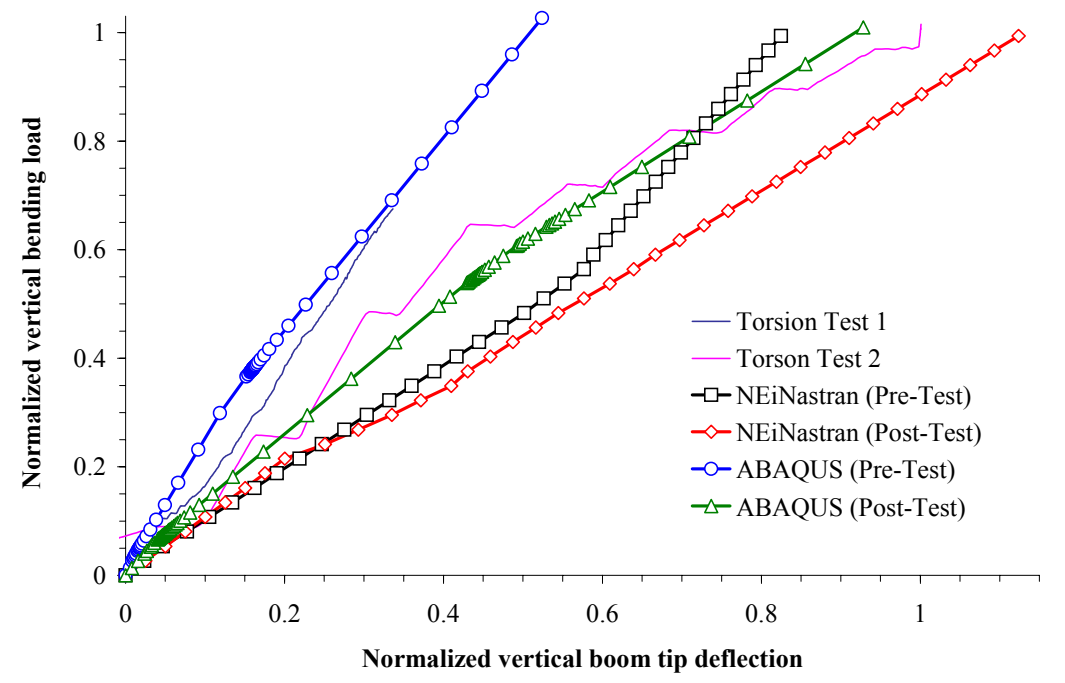

Figure 19. Combined vertical bending and torsion test and analysis results for 10-m beam structural assembly static load-deflection test. 
The first three vibration frequencies of the beam structural assembly test and FEA post-test predictions are listed in Table 3. The FEA mode shapes were selected based on the resemblance to the modes shapes of the test. The results of both FE models using the reduced properties were in reasonable agreement for the first three frequencies and mode shapes. As previously mentioned, contributing factors that affected the test/analysis correlation were uncertainties in the stiffness in the spreader system lines. Also, ignoring the modeling of the gravity-offload system may have contributed to errors in the predicted frequencies.

Table 3. Comparison of test and FEA frequencies for 10-m beam structural assembly vibration test.

\begin{tabular}{|c|c|c|c|}
\hline Mode & Test Frequency $(\mathrm{Hz})$ & NEiNastran Frequency $(\mathrm{Hz})$ & ABAQUS Frequency $(\mathrm{Hz})$ \\
\hline 1 & 1.38 & $0.87(-37.0 \%)$ & $1.20(-15.0 \%)$ \\
\hline 2 & 4.65 & $4.71(+1.3 \%)$ & $3.59(-22.8 \%)$ \\
\hline 3 & 9.00 & $7.79(-13.4 \%)$ & $7.28(-19.1 \%)$ \\
\hline
\end{tabular}

\section{Concluding Remarks}

Validated finite element methods and models are needed to provide accurate predictions of the structural response of future solar sail missions. A comprehensive phased test plan has been performed to permit validation of FEA models simulating the response of sub-scale solar sail ground tests. These tests consist of solar sail component, subsystem, and sub-scale system ground tests that simulate the vacuum and thermal conditions of the space environment. Recently, two solar sail test articles, a 7.4-m beam assembly subsystem test article and a 10-m fourquadrant solar sail system test article, were tested in vacuum conditions with gravity-offload systems to mitigate the effects of gravity. This paper presented the structural analyses that simulated the ground tests using the finite element analysis software packages, NEiNastran and ABAQUS, and the correlation of the analyses with the test results. Comparisons were made for pre-test and post-test analysis predictions from both software packages with the test data including load-deflection curves from static load tests and vibration frequencies and mode shapes from vibration tests. The finite element analysis predictions for the 7.4-m beam assembly were in reasonable agreement for the vertical bending test and in poor agreement for the structural vibration test. The finite element predictions for the 10-m four-quadrant solar sail test article were in reasonable agreement for the static loads tests and in good agreement for the structural vibration test. Contributing factors affecting the test/analysis correlation in both tests were uncertainties in unmeasured material properties and conditions during the tests such as the stiffness of the spreader system lines. In addition, the modeling assumption to simulate the tests without the gravity-offload system also contributed to the difference between the test and analysis predictions. Overall, the finite element analysis predictions using NEiNastran and ABAQUS were in reasonable agreement with the solar sail ground test data considering the complexity of the test set-up. Moreover, the correlation of the analyses with the test data should improve with additional analyses to calibrate the models and a better understanding of the test set-up and loading conditions. The knowledge gained in this validation effort will be used in the modeling and structural analyses of a 20-m solar sail system test to be performed at the Space Power Facility located at the NASA Glenn Research Center Plum Brook Station in July 2005. The focused efforts of this project in the In-Space Propulsion Technology Program will advance solar sail technology needed for a solar sail flight demonstration.

\section{Acknowledgments}

The work described in this paper was funded in whole or in part by the In-Space Propulsion Technology Program, which is managed by NASA's Science Mission Directorate in Washington, D.C., and implemented by the In-Space Propulsion Technology Office at Marshall Space Flight Center in Huntsville, Alabama. The program objective is to develop in-space propulsion technologies that can enable or benefit near and mid-term NASA space science missions by significantly reducing cost, mass or travel times. 


\section{References}

${ }^{1}$ Wright, J., Space Sailing, Taylor and Francis Publishers, 1992.

${ }^{2}$ McInnes, C. R., Solar Sailing: Technology, Dynamics, and Mission Applications, Springer-Praxis Series in Space Science and Technology, 1999.

${ }^{3}$ Chmielewski, A. B., "Advanced Concepts," Gossamer Spacecraft: Membrane and Inflatable Structures Technology for Space Applications, Edited by C.H.M. Jenkins, Progress in Astronautics and Aeronautics, Vol. 191, American Institute of Aeronautics and Astronautics, Reston, VA, 2001.

${ }^{4}$ Salama, M., McInnes, C. R., and Mulligan, P., "Gossamer Spacecraft Technology," Gossamer Spacecraft: Membrane and Inflatable Structures Technology for Space Applications, Edited by C.H.M. Jenkins, Progress in Astronautics and Aeronautics, Vol. 191, American Institute of Aeronautics and Astronautics, Reston, VA, 2001.

${ }^{5}$ West, J. L. and Derbès, B., "Solar Sail Vehicle System Design for the Geostorm Warning Mission," $41^{s t}$ AIAA/ASME/ASCE/AHS/ASC Structures, Structural Dynamics \& Materials Conference, AIAA-2000-5326, 2000.

${ }^{6}$ West, J. L., "The Geostorm Warning Mission: Enhanced Opportunity Based on New Technology," 14th AAS/AIAA Space Flight Mechanics Conference, AAS 2004-102, 2004.

${ }^{7}$ Lichodziejewski, D., Derbès, B., West, J., Reinert, R., Belvin, K., and Pappa, R., "Bringing an Effective Solar Sail Design Toward TRL 6", 39th AIAA/ASME/SAE/ASEE Joint Propulsion Conference and Exhibit, AIAA 2003-4659, 2003.

${ }^{8}$ Lichodziejewski, D., Derbès, B., Reinert, R., Belvin, K., Slade, K., and Mann, T., "Development and Ground Testing of a Compactly Stowed Scalable Inflatably Deployed Solar Sail," $45^{\text {th }}$ AIAA/ASME/ASCE/AHS/ASC Structures, Structural Dynamics \& Materials Conference, AIAA-2004-1507, 2004.

${ }^{9}$ NEiNastran User's and Reference Manual, Version 8.3, Noran Engineering, Inc, 2004.

${ }^{10}$ ABAQUS/Standard User's Manual, Version 6.4-2, ABAQUS, Inc., 2004.

${ }^{11}$ Lichodziejewski, D., Derbès, B., Slade, K., and Mann, T., "Vacuum Deployment and Testing of a 4-Quadrant Scalable Inflatable Rigidizable Solar Sail System," $46^{\text {th }}$ AIAA/ASME/ASCE/AHS/ASC Structures, Structural Dynamics \& Materials Conference, AIAA 2005-2122, 2005. 